\title{
Curcumin Protects Neurons from Glutamate-Induced Excitotoxicity by Membrane Anchored AKAP79-PKA Interaction Network
}

\author{
Kui Chen, ${ }^{1,2}$ Yu An, ${ }^{1,2}$ Lu Tie, ${ }^{1,2}$ Yan Pan, ${ }^{1,2}$ and Xuejun $\mathrm{Li}^{1,2}$ \\ ${ }^{1}$ State Key Laboratory of Natural and Biomimetic Drugs, Department of Pharmacology, School of Basic Medical Sciences, \\ Peking University, Beijing 100191, China \\ ${ }^{2}$ Beijing Key Laboratory of Tumor Systems Biology, Peking University, Beijing 100191, China
}

Correspondence should be addressed to Xuejun Li; xjli@bjmu.edu.cn

Received 31 January 2015; Revised 17 May 2015; Accepted 25 May 2015

Academic Editor: David Mischoulon

Copyright (C) 2015 Kui Chen et al. This is an open access article distributed under the Creative Commons Attribution License, which permits unrestricted use, distribution, and reproduction in any medium, provided the original work is properly cited.

\begin{abstract}
Now stimulation of AMPA receptor as well as its downstream pathways is considered as potential central mediators in antidepressant mechanisms. As a signal integrator which binds to AMPA receptor, A-kinase anchoring protein 79-(AKAP79-) PKA complex is regarded as a potential drug target to exert neuroprotective effects. A well-tolerated and multitarget drug curcumin has been confirmed to exert antidepressant-like effects. To explore whether AKAP79-PKA complex is involved in curcumin-mediated antiexcitotoxicity, we detected calcium signaling, subcellular location of AKAP79-PKA complex, phosphorylation of glutamate receptor, and ERK and AKT cascades. In this study, we found that curcumin protected neurons from glutamate insult by reducing $\mathrm{Ca}^{2+}$ influx and blocking the translocation of AKAP79 from cytomembrane to cytoplasm. In parallel, curcumin enhanced the phosphorylation of AMPA receptor and its downstream pathways in PKA-dependent manner. If we pretreated cells with PKA anchoring inhibitor $\mathrm{Ht} 31$ to disassociate PKA from AKAP79, no neuroprotective effects were observed. In conclusion, our results show that AKAP79-anchored PKA facilitated the signal relay from AMPA receptor to AKT and ERK cascades, which may be crucial for curcumin-mediated antiexcitotoxicity.
\end{abstract}

\section{Introduction}

To clarify the pathogenesis of major depressive disorder (MDD) and increase effective rate of antidepressant treatment is the direction of all the psychiatrists in the world. Today, the situation of antidepressant treatment is not optimistic, because the response rate of current antidepressants is just $60-70 \%$ and the clinical remission rate is only about $30 \%$ which suggests the imbalance of monoamine neurotransmitters may not be the key pathogenesis for MDD [1]. Currently, the orientation of antidepressant development is mainly focused on NMDA receptor antagonists due to its rapid and long-lasting antidepressant effects [2]. Blocking NMDA receptors can not only inhibit excessive glutamatemediated activation of extrasynaptic NMDA receptor but also enhance AMPA receptor signal transduction to exert antidepressant effects [3]. In view of the popular abnormal glutamate receptors hypothesis, glutamate-induced excitotoxicity is increasingly used as cell model of MDD, which is characterized by glutamate receptor excessive activation and calcium overload [4-6].

Interestingly, cAMP-PKA cascade has been reported to associate with pathophysiology of MDD and ketaminemediated antidepressant actions [7]. Reduced PKA activity has been observed in depressed patients and antidepressants can upregulate PKA activity [8]. In vitro studies also found that PKA activators showed the antidepressant-like effects in animal model of depression, while PKA activators mediated antidepressant effects could be totally blocked by PKA inhibitor, suggesting PKA may serve as a new drug target for depression treatment $[9,10]$. The A-kinase anchoring proteins (AKAPs) are signal-assembling hub which can target 
various enzymes in the appropriate compartment. Notably, AKAPs have high affinity to the regulatory subunit of PKA and anchor PKA in the precise subcellular location. In the brain, AKAP79 is the main AKAP subunit which can direct PKA toward AMPA receptor subunit GluR1 in neuronal postsynaptic membrane. So theoretically, it is reasonable to speculate that AKAP79-PKA complex may be involved in the antidepressant mechanisms of NMDAR antagonists or PKA enhancers.

As a polyphenolic natural product, curcumin has already been confirmed to have antiexcitotoxicity effects [11]. In addition, curcumin has also been demonstrated to show antidepressant-like effects in MDD animal models, which are exposed to chronic unpredictable mild stress (CUMS) [12]. Considering that curcumin has a range of drug targets and can influence numerous signal transmissions, to find the specific and key mechanism of curcumin-mediated antidepressant effects is extremely urgent. In this paper, we employed $\mathrm{SH}$ SY5Y human neuroblastoma cells as the experimental model for glutamate excitotoxicity, and all experiments reported here were designed to evaluate whether AKAP79-PKA complex participated in curcumin-mediated neuroprotective effects as the crucial molecular mechanism.

\section{Materials and Methods}

2.1. Reagents. Curcumin and glutamate were purchased from Sigma-Aldrich (St. Louis, MO, USA). The LDH assay kit was from Nanjing Jiancheng Bioengineering Institute (Nanjing, China). Antibodies to MEK1/2, p-MEK1/2, ERK1/2, pERK1/2, AKT, and p-AKT were obtained from Cell Signaling Technology (Beverly, MA, USA). Antibodies to PKA, NR1, p-NR1, GluR1, p-GluR1, GAPDH, $\mathrm{Na}^{+}-\mathrm{K}^{+}$-ATPase $\alpha 1$, and rabbit IgG were purchased from Bioworld (Louis Park, MN, USA). AKAP79 antibody and Protein A/G-Agarose were from Santa Cruz Biotechnology (Santa Cruz, CA, USA). Alkaline phosphatase-conjugated secondary antibodies were purchased from PIERCE. BCIP (3-bromo-4-chloro-5-indolyl phosphate) and NBT (nitro blue tetrazolium) were obtained from Ameresco. DyLight 488 and DyLight 594 conjugated secondary antibodies were bought from Jackson ImmunoResearch Laboratories (West Grove, PA, USA). The MTS assay kit was bought from Promega. The calcium indicator dye fluo-3 was bought from Beyotime Biotechnology. The BCA assay kit and chamber coverslip were purchased from Thermo Fisher Scientific (Rockford, IL). Fetal bovine serum was from HyClone (Logan, UT). Protease inhibitor cocktails were purchased from Roche (Basel, Switzerland).

2.2. Drug Treatment. Curcumin was dissolved in dimethyl sulfoxide (DMSO, $\leq 0.01 \%)[13,14]$. The PKA anchoring inhibitor Ht31 and control fragment Ht31p (both $20 \mu \mathrm{M}$ ) were pretreated for $45 \mathrm{~min}$ before the administration of curcumin $[15,16]$. Glutamate was dissolved in Locke's buffer $\left(154 \mathrm{mM} \mathrm{NaCl}, \quad 5.6 \mathrm{mM} \mathrm{KCl}, 3.4 \mathrm{mM} \mathrm{NaHCO}_{3}\right.$, $1.2 \mathrm{mM} \mathrm{MgCl}_{2}, \quad 5.6 \mathrm{mM}$ glucose, $5 \mathrm{mM}$ HEPES, and $2.3 \mathrm{mM} \mathrm{CaCl}_{2}, \mathrm{pH}$ 7.4) [4]. Neurons were exposed to $30 \mathrm{mM}$ glutamate for 12 hours to induce excitotoxicity.
2.3. Measurement of Intracellular Free $\mathrm{Ca}^{2+}$ Concentration. Intracellular free $\mathrm{Ca}^{2+}$ levels $\left(\left[\mathrm{Ca}^{2+}\right]_{i}\right)$ were quantified by fluorescence imaging of the calcium indicator dye fluo-3. Cells were incubated for $30 \mathrm{~min}$ in the presence of $5 \mu \mathrm{M}$ fluo-3 and then washed twice to remove extracellular dye. Cells were further incubated for $30 \mathrm{~min}$ prior to imaging. The fluorescence intensity was monitored by Zeiss microscope. The $\left[\mathrm{Ca}^{2+}\right]_{i}$ in 10-15 neuronal cell bodies per microscope field was monitored prior to and after exposure of cells to glutamate insult.

2.4. Cell Culture. SH-SY 5Y cells were purchased from the Cell Culture Centre of Institute of Basic Medical Science, Chinese Academy of Medical Sciences. Briefly, the cells were cultured in DMEM medium (high glucose, no glutamine) supplemented with $2 \mathrm{mM}$ L-glutamine, fetal bovine serum $10 \%$ (growth medium for cell proliferation) or $2.5 \%$ (maintenance medium for drug treatment, glutamate insult, and follow-up experiments), and antibiotics (penicillin and streptomycin) at $37^{\circ} \mathrm{C}$ in a humidified atmosphere consisting of $95 \%$ air $/ 5 \% \mathrm{CO}_{2}$. Upon reaching $75 \%$ confluence, the cells were exposed to either drugs or vehicle and then collected and extracted by RIPA lysis buffer (50 mM Tris- $\mathrm{HCl}$ ( $\mathrm{pH} 7.4$ ), $150 \mathrm{mM} \mathrm{NaCl}, 1 \% \mathrm{NP}-40$, and $0.1 \%$ SDS) containing 1\% PMSF and protease inhibitor cocktails. The lysates were centrifuged at $12,000 \mathrm{rpm}$ for 20 minutes at $4^{\circ} \mathrm{C}$. The supernatants were collected and stored at $-80^{\circ} \mathrm{C}$. Sample protein concentrations were determined by BCA assay.

2.5. MTS Assay. SH-SY 5Y cells were plated in 96-well plates for 24 hours. In accordance with our previous research, pretreatment of $1 \mu \mathrm{M}$ curcumin for 6 hours was employed in the present study and $30 \mathrm{mM}$ glutamate was incubated with neurons for 12 hours $[4,17,18]$. After incubation with drugs, cell viabilities were determined by MTS (3-(4,5-dimethylthiazol-2-yl)-5-(3-carboxymethoxyphenyl)-2-(4-sulfophenyl)-2H-tetrazolium, inner salt) assay. After three washes with PBS, the cells were exposed to $100 \mu \mathrm{L}$ new media containing $20 \mu \mathrm{L} \mathrm{MTS}$ at $37^{\circ} \mathrm{C}$ for 3 hours. The absorbance was measured at $490 \mathrm{~nm}$ using a 96 -well plate reader.

2.6. $\mathrm{LDH}$ Assay. In the lactate dehydrogenase ( $\mathrm{LDH})$ assay, cell injury was evaluated by measuring the leakage of LDH from impaired neurons. LDH activities in cell culture supernatants were examined as previously described [19]. The amounts of released LDH were determined by measuring the absorbance at a wavelength of $492 \mathrm{~nm}$.

2.7. Western Blot Analysis. Equal amounts of protein extracts were loaded and separated by 5-12\% SDS-PAGE gel and transferred onto PVDF membranes. After blocking with 5\% skimmed milk, the membranes were incubated with the primary antibodies at $4^{\circ} \mathrm{C}$ overnight. Then alkaline phosphataseconjugated secondary antibodies were adopted before treatment with the BCIP/NBT substrate solution. The western blot intensities were subsequently analyzed by Image J software.

2.8. Subcellular Fractionation. To determine the subcellular distribution of the AKAP79 in the cytoplasm and membrane 
fractions, cell lysates were prepared by ice-cold lysis buffer (10 mM Tris base, pH 7.6, $320 \mathrm{mM}$ sucrose, $150 \mathrm{mM} \mathrm{NaCl}$, $5 \mathrm{mM}$ EDTA, $5 \mathrm{mM}$ EGTA, $1 \mathrm{mM}$ benzamidine, $1 \mathrm{mM} 4$ (2-aminoethyl) benzenesulfonyl fluoride, $2 \mathrm{~g} / \mathrm{mL}$ leupeptin, $2 \mathrm{~g} / \mathrm{mL}$ pepstatin, and $50 \mathrm{mM} \mathrm{NaF}$ ) followed by homogenization. The homogenates were centrifuged at $960 \times \mathrm{g}$ to remove nuclei and large debris (P1). The supernatants (S1) were fractionated into crude synaptosomal membranes (P2) and soluble supernatant/cytoplasm (S2) by $10,000 \times \mathrm{g}$ for $30 \mathrm{~min}$. The P2 pellet was homogenized in lysis buffer as above; aliquots of P2 and S2 were taken for western analysis [20].

2.9. Coimmunoprecipitation. Proteins were isolated from the cultured cells and $1000 \mu \mathrm{g}$ supernatants were transferred into each of microcentrifuge tubes. For preclearing the lysates, $1.0 \mu \mathrm{g}$ of control IgG and $20 \mu \mathrm{L}$ of Protein A/G-Agarose were added. Then $2 \mu \mathrm{g}$ of anti-AKAP79 primary antibody was placed in the tubes and incubated at $4^{\circ} \mathrm{C}$ overnight before the addition of $20 \mu \mathrm{L}$ of Protein A/G-Agarose. The mixture was incubated at $4^{\circ} \mathrm{C}$ on a rotating device overnight. The immunoprecipitates were collected by centrifugation and the pellets were gently washed with $1.0 \mathrm{~mL}$ RIPA buffer for twice to four times. The final pellets were resuspended with $40 \mu \mathrm{L}$ of $2 \mathrm{x}$ electrophoresis sample buffer and boiled for 5 minutes. Finally, the proteins were analyzed by western blot.

2.10. Immunofluorescence Assay. SH-SY $5 Y$ cells were plated on chamber coverslips. After drug treatment, the chamber coverslips were gently washed with PBS, fixed in $3.7 \%$ paraformaldehyde for $15 \mathrm{~min}$, permeabilized in PBS containing $0.1 \%$ Triton X-100 for $5 \mathrm{~min}$, and blocked in PBS containing $5 \%$ BSA for $30 \mathrm{~min}$. The fixed cells were incubated with antiAKAP79 and anti-PKA RII antibodies overnight at $4^{\circ} \mathrm{C}$ and incubated with the DyLight 488 and DyLight 594 conjugated secondary antibodies for $2 \mathrm{~h}$. After PBS wash, nucleus was stained by Hoechst 33342 for $10 \mathrm{~min}$. The images were recorded by TCS-SP5 laser scanning confocal microscope (Leica Microsystems, Germany).

2.11. Statistical Analysis. The data shown were obtained from at least three independent experiments. The data in the different experimental groups are expressed as the mean \pm S.E.M. Differences between the groups were evaluated by one-way ANOVA followed by Dunnett's test or Student-NewmanKeuls multiple comparison tests when necessary. Significant differences were represented as ${ }^{*} P<0.05,{ }^{* *} P<0.01$, or ${ }^{* * *} P<0.001$.

\section{Results}

3.1. Pretreatment with Curcumin Attenuated Glutamate-Mediated Calcium Influx and Inhibited AKAP79 Translocation from Membrane to Cytoplasm. In this experiment, we examined $\left[\mathrm{Ca}^{2+}\right]_{i}$ in control group, glutamate group, curcuminpretreated group, and MK-801 group. The basal concentration of intracellular free $\mathrm{Ca}^{2+}$ was similar in all groups. In glutamate group, glutamate induced an obvious and long-term increase of calcium concentration. Unlike glutamate group, both the amplitude and the duration of $\mathrm{Ca}^{2+}$ influx were significantly attenuated in neurons of curcumin-pretreated group. Similarly, no calcium influx was observed in MK801 pretreatment group (Figure 1(a)). In order to study the influence of calcium influx on the translocation of AKAP79PKA complex, subcellular fractionation assay was conducted. As shown in Figures 1(b)-1(d), less AKAP79 was targeted to the plasma membrane after glutamate insult $(F(3,8)=14.39$, $P=0.0014)$, while curcumin inhibited the redistribution of AKAP79-PKA complex from membrane to cytoplasm $(F(3,8)=10.52, P=0.0038)$.

3.2. Curcumin Strengthened AKAP79-PKA Interaction Even after Glutamate Exposure. Coimmunoprecipitation assay showed that glutamate apparently attenuated the interaction between AKAP79 and PKA (Dunnett's test, $q=2.989, P<$ $0.05)$, suggesting that glutamate was able to disturb the precise subcellular location of AKAP79-anchored PKA (Figures 2(a) and 2(b)). However, curcumin obviously strengthened AKAP79-PKA interaction (Dunnett's test, $q=15.08, P<$ 0.001 ) even after glutamate exposure (Dunnett's test, $q=$ $6.259, P<0.001$ ) (Figures 2(a) and 2(b)). Immunofluorescence assay also found similar results for AKAP79-PKA interaction in membrane (Control versus Glu: Dunnett's test, $q=$ 2.986, $P<0.05$; Control versus Cur: Dunnett's test, $q=6.236$, $P<0.001$; Control versus Cur + Glu: Dunnett's test, $q=$ 3.294, $P<0.05)$ (Figure 2(c)).

3.3. Curcumin Facilitated the Phosphorylation of AMPA Receptor but not NMDA Receptor. We further explored whether curcumin could influence the phosphorylation of AMPA receptor subunit GluR1 and NMDA receptor subunit NR1. No significant change was observed in p-NR1 $(F(5,12)=2.878$, $P=0.062$ ), while $\mathrm{p}-\mathrm{GluR} 1$ level is enhanced for a long time especially after curcumin exposure for 6 hours $(F(5,12)=$ 5.531, $P=0.0072$ ) (Figure 3).

3.4. AKAP-Anchored PKA Was Crucial for Curcumin-Induced Activation of ERK and AKT Cascade. Figures 4(a)-4(e) showed that phosphorylation of AKT (SNK test, $q=9.166$, $P<0.001$ ), MEK1/2 (SNK test, $q=9.766, P<0.001$ ), ERK1/2 (SNK test, $q=5.136, P<0.05$ ), and CREB (SNK test, $q=$ 9.752, $P<0.001$ ) was enhanced after curcumin treatment for 6 hours, while disruption of PKA anchoring with Ht31 attenuated curcumin-induced activations of ERK1/2 and AKT cascades.

As AKAP-anchored PKA was pivotal for neuronal survival related signaling pathway, we next performed experiments to determine whether curcumin-mediated neuroprotection was blocked by PKA anchoring inhibitor Ht31. MTS and $\mathrm{LDH}$ results showed that SH-SY 5Y neuronal cells presented a remarkable decrease in cell viability (Dunnett's test, $q=4.927, P<0.01$ ) and an increased LDH release (Dunnett's test, $q=5.457, P<0.001)$ after exposure to $30 \mathrm{mM}$ glutamate over 12 hours, while $1 \mu \mathrm{M}$ curcumin treatment for 6 hours displayed the effectively neuroprotective effect against glutamate excitotoxicity (MTS: Dunnett's test, $q=1.518, P>0.05$; LDH: Dunnett's test, $q=0.467, P>0.05$ ) (Figures $4(\mathrm{f})$ and 


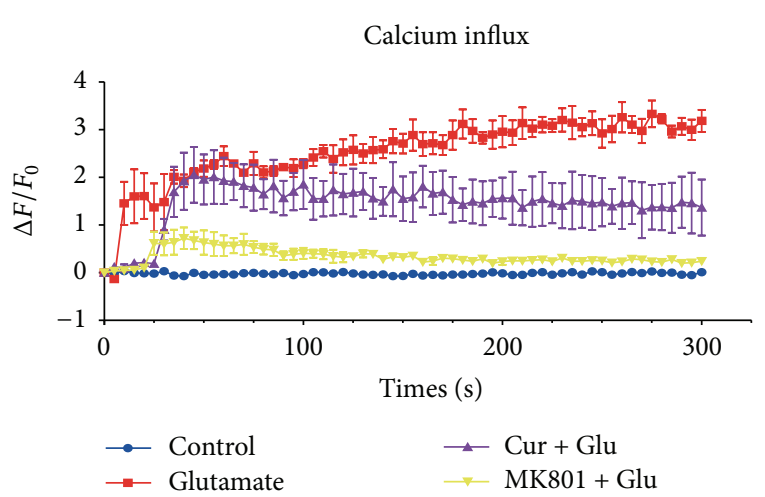

(a)

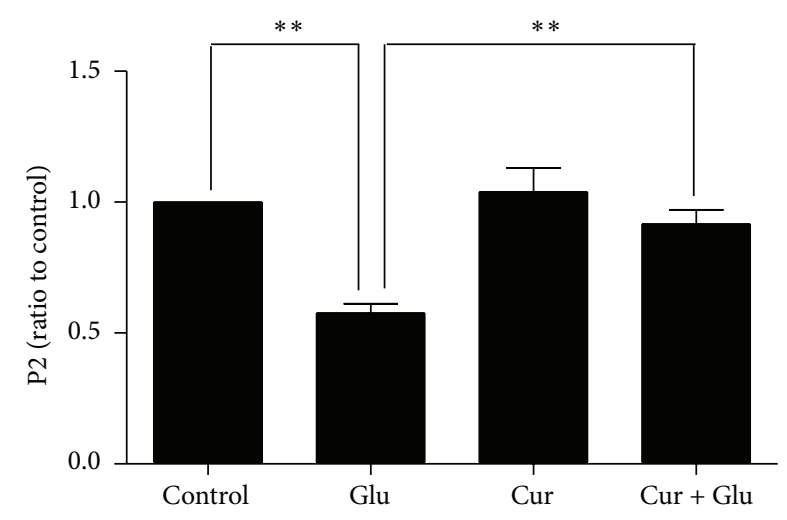

(c)

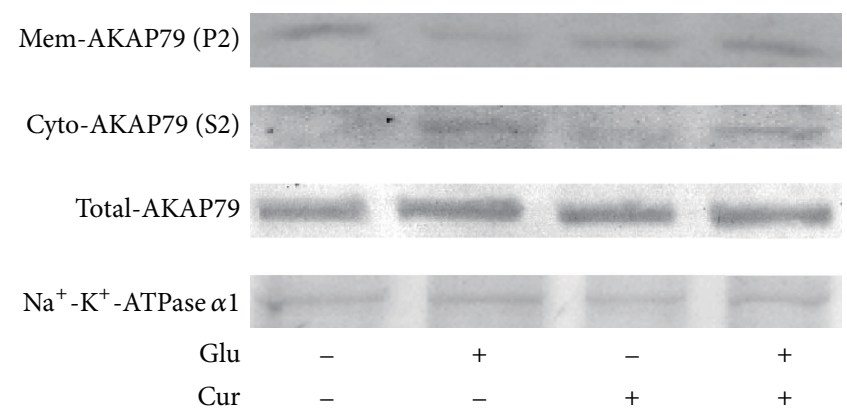

(b)

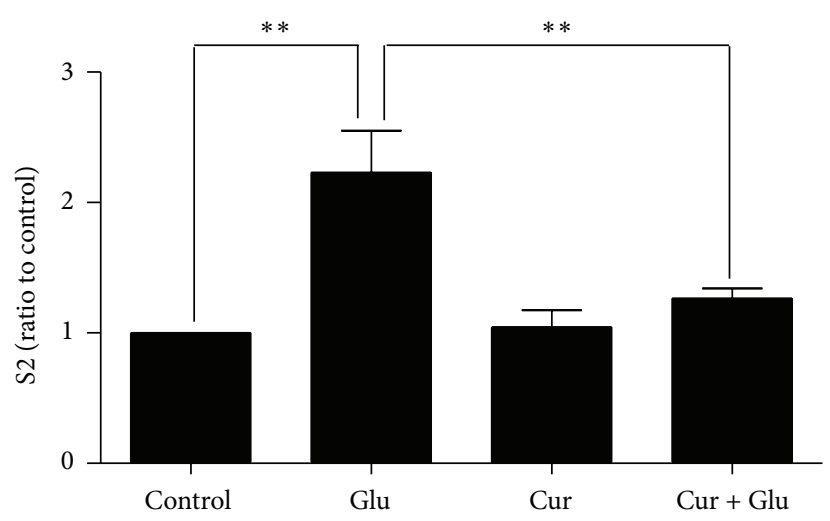

(d)

FIGURE 1: Curcumin blocked glutamate-mediated calcium influx and blocked the translocation of AKAP79-PKA complex. (a) Summary data describing the change of $\left[\mathrm{Ca}^{2+}\right]_{i}$. (b) Glutamate induced an increase of AKAP79 in the S2 cytosolic fraction accompanied by decrease of AKAP79 in the P2 membrane fraction, which was blocked by curcumin. For membrane protein detection, commonly used internal reference protein is $\mathrm{Na}^{+}-\mathrm{K}^{+}$-ATPase. (c, d) Western blotting bands were quantified using Image J software. ${ }^{* *} P<0.01$ versus glutamate group. $(n=3)$.

$4(\mathrm{~g}))$. Notably, no neuroprotective effect of the curcumin was observed if we administrated PKA anchoring inhibitor Ht31 in advance (MTS: Dunnett's test, $q=3.507, P<0.05$; LDH: Dunnett's test, $q=4.428, P<0.01$ ) (Figures $4(\mathrm{f})$ and $4(\mathrm{~g})$ ).

\section{Discussion}

In the present study, we have demonstrated that persistent and excessive glutamate insults triggered $\mathrm{Ca}^{2+}$ influx through NMDA receptor in SH-SY5Y human neuroblastoma cells, which is the key toxic mechanism of neurologic diseases, such as MDD, brain trauma, and Alzheimer's disease [21, 22]. To prove calcium response to glutamate was indeed mediated by NMDA receptor, we pretreated cells with NMDA receptor antagonist $\mathrm{MK}-801$ and then exposed neurons to glutamate insult. Results showed that no calcium influx was observed in MK-801 pretreatment group. Similar to MK801, curcumin also suppressed calcium overload in order to maintain cellular homeostasis for neuronal survival, which suggested that curcumin could antagonize NMDA receptor and block NMDA receptor-mediated calcium influx.
Recently, it has been presumed that PKA plays a critical role in antiexcitotoxic and antidepressant mechanisms [9, $10]$, and AKAP is the dominator to regulate the spatial and temporal organization of PKA as well as PKA related signal pathways [23]. As a main subunit of AKAP in SH-SY5Y neurons, scaffold protein AKAP79 is targeted to AMPA receptor in the postsynaptic density by interactions with cytoskeleton actin and PSD-95. Our study provides evidence that inhibition of $\mathrm{Ca}^{2+}$ influx by curcumin contributes to maintaining the membrane anchoring of AKAP79-PKA complex, and our results are in line with previous findings that blocking NMDA receptor-mediated calcium influx is in favor of maintaining the association of AKAP79 with membrane and AMPA receptor subunit GluR1 [20]. Increased $\left[\mathrm{Ca}^{2+}\right]_{i}$ has been reported to induce remodeling of cytoskeleton and trigger the redistribution of signaling proteins bound to cytoskeleton from postsynaptic membranes to cytoplasm after glutamate insult [20]. As Smith reported [20], glutamate disrupted the precise anchoring of AKAP79-PKA compound leading to AKAP79 loss from postsynaptic membrane in our study. Unexpectedly, glutamate also reduced AKAP79PKA interaction to target even less PKA around AMPA 


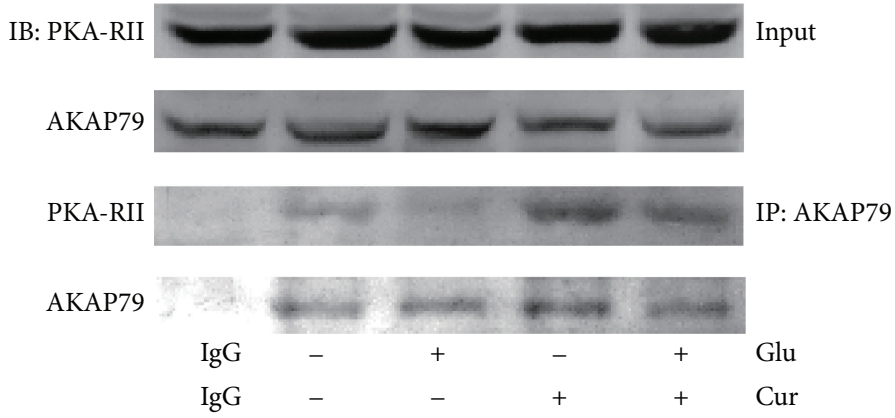

(a)
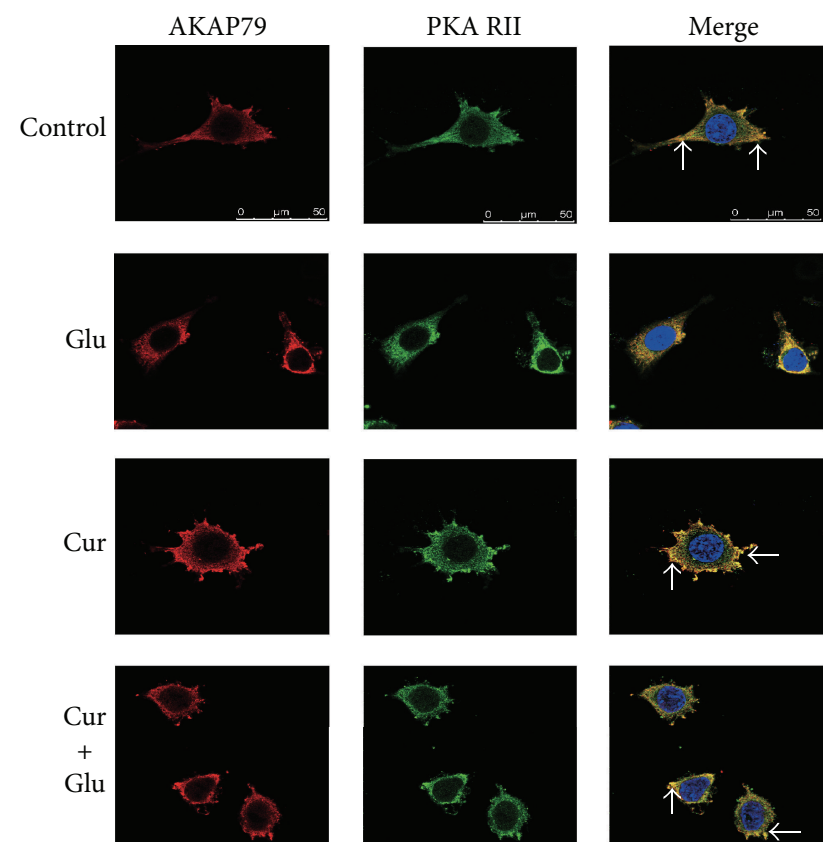

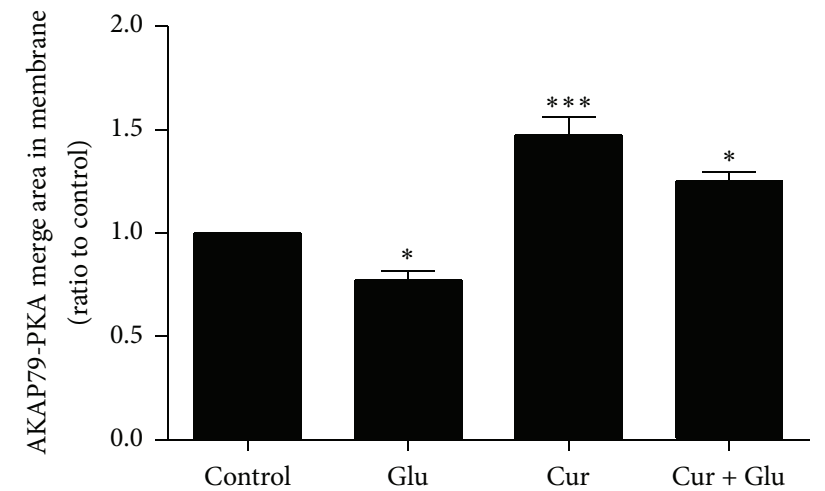

(c)

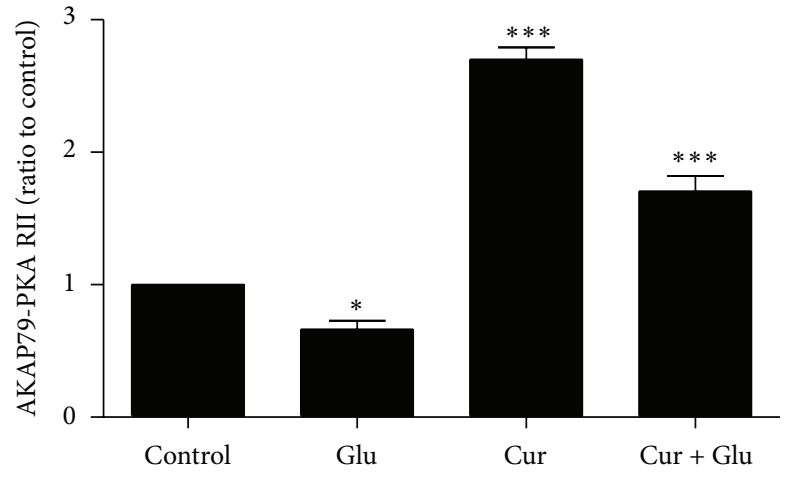

(b)

FIgURE 2: Curcumin enhanced the interaction between AKAP79 and PKA at the plasma membrane. (a) The change of AKAP79-PKA interaction after exposure to curcumin with or without glutamate exposure. (b) Western blotting bands were quantified using Image J software. (c) The colocalization changes between AKAP79 and PKA in membrane. The red fluorescent indicated the AKAP79 staining and the green fluorescent showed the PKA staining, while nucleus stained by Hoechst 33342 emitted blue fluorescent. The red fluorescent and green fluorescent merged into yellow staining which suggested the overlap between AKAP79 and PKA. Scale bar, $50 \mu \mathrm{m}$. The colocalization of AKAP79 and PKA was quantified using Image J software. ${ }^{*} P<0.05$ and ${ }^{* * *} P<0.001$ versus control values. $(n=3)$.

receptor. On the contrary, curcumin retained more AKAP79PKA complex on the neuronal membrane and augmented AKAP79-PKA interaction.

What is the significance of targeting more AKAP79 compounds around GluR1 and enhancing AKAP79-PKA interaction after curcumin treatment? AKAP79-anchored PKA is reported to phosphorylate GluR1 to increase the open probability of AMPA receptor and further relay signals to downstream pathways via PI3K-dependent activation of MAPK $[24,25]$. After AKAP79/150 knockout, Ser-845 phosphorylation of GluR1 is significantly reduced which highlights the strong impact of AKAP79/150 and AKAP-anchored PKA on the function of GluR1 [26]. Thus, we hypothesized that curcumin could influence the phosphorylation of GluR1 or NR1. As we expected, increased phosphorylation of the GluR1 was found after $1 \mu \mathrm{M}$ curcumin pretreatment, while there was no remarkable difference for p-NR1, which suggested that enhanced phosphorylated GluR1 may participate in curcumin-mediated neuroprotective process.

AMPA receptor-mediated upregulation of AKT and ERK cascades is reported to be crucial for NMDA receptor antagonist-induced rapid antidepressant effects [27], so we further investigated whether ERK and AKT cascades were involved in curcumin-mediated neuroprotective effects. 


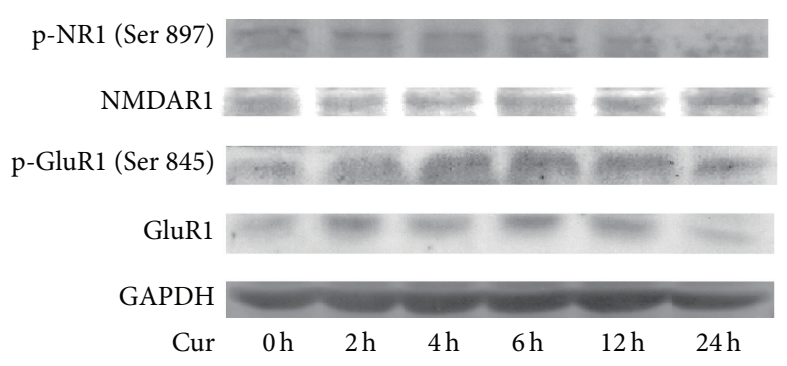

(a)

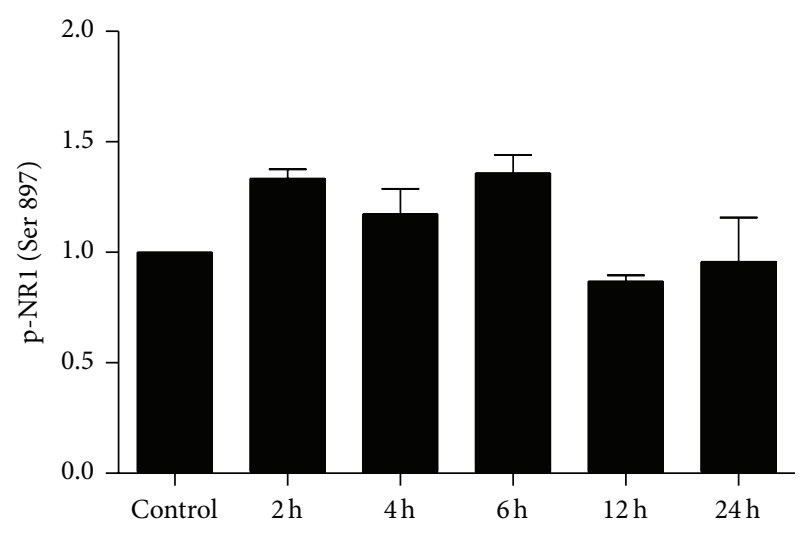

(b)

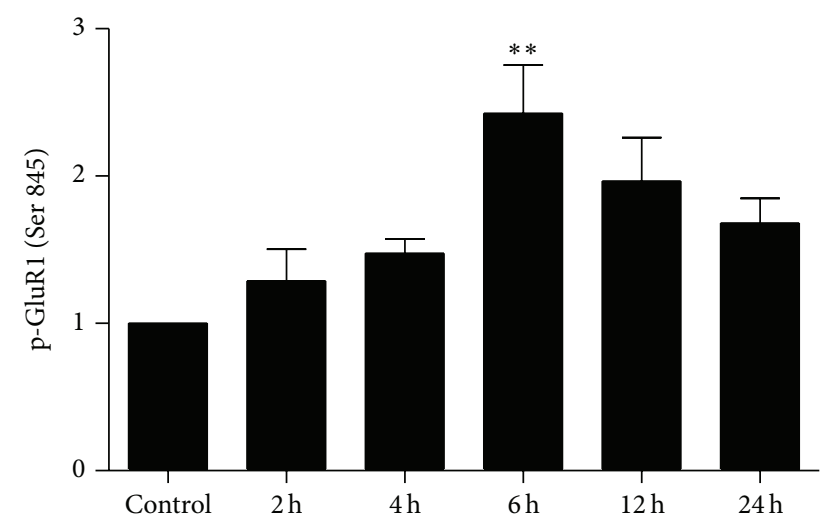

(c)

FIGURE 3: The time-course of curcumin on the phosphorylations of NR1 and GluR1. (a) Curcumin facilitated the phosphorylation of GluR1. GAPDH serves as the commonly used internal reference protein. $(b, c)$ Western blotting bands were quantified using Image J software. ${ }^{* *} P<0.01$ versus control values. $(n=3)$.

Enhanced AKT and ERK cascades were observed after curcumin treatment. Importantly, the signal transmitted by AKT and ERK cascades further resulted in upregulation of $\mathrm{p}$ CREB. CREB is a cellular transcription factor which binds to cAMP response elements (CRE) sequence of DNA to manipulate the transcription of the downstream genes, such as BDNF gene. CREB phosphorylation at serine 133 is important for the survival of neurons [28]. Interestingly, activation of AKT and ERK cascades is in PKA-dependent manner. If we pretreated neurons with PKA anchoring inhibitor Ht31, no activation of ERK1/2 and AKT cascades was observed. Accordingly, MTS and LDH assays confirmed that curcumin did rescue neurons from glutamate insult in PKA-dependent manner which indicated that AKAP79-anchored PKA is crucial for curcumin to resist glutamate insult in MDD cell models. Our results are consistent with previous findings that AKAP-anchored PKA can facilitate signal relay from MEK1/2 to ERK1/2 [29]. Additionally, our observations are also in line with the evidence that PKA is required for AKT phosphorylation [30]. It should be noted that $\mathrm{Ht} 31$ is a nonselective PKA anchoring inhibitor; other AKAP subunits may also be involved in curcumin-mediated neuroprotective effects.
In conclusion, the present study provides direct evidence that AKAP79-PKA complex participates in the antidepressant mechanisms of curcumin. Targeting more AKAP79anchored PKA to membrane is in favor of activating GluR1 and its downstream pathways, such as AKT and ERK cascades. Thus AKAP79-PKA complex can serve as potential drug targets to regulate anchored signaling protein and their downstream pathways, which have implications for research and development of novel antiexcitotoxic and antidepressant drugs.
Abbreviations
MDD:
Major depressive disorder
NMDA receptor: N-Methyl-D-aspartic acid receptor
AMPA receptor: $\alpha$-Amino-3-hydroxy-5-methyl-4- isoxazolepropionic acid receptor
AKAP: A-kinase anchoring proteins
PKA: $\quad$ Protein kinase A
CUMS: $\quad$ Chronic unpredictable mild stress 


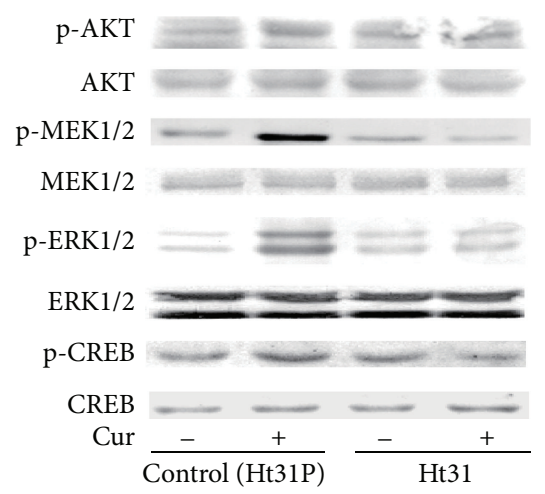

(a)

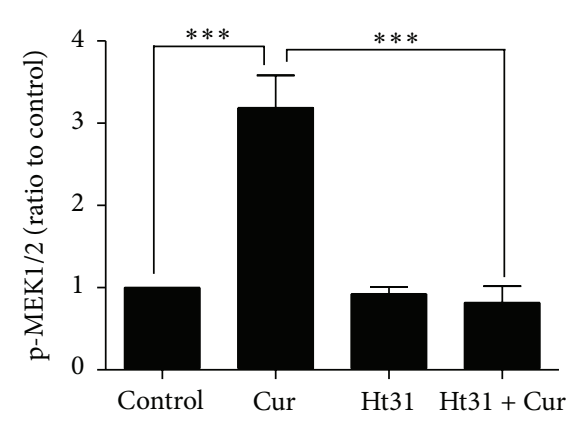

(c)

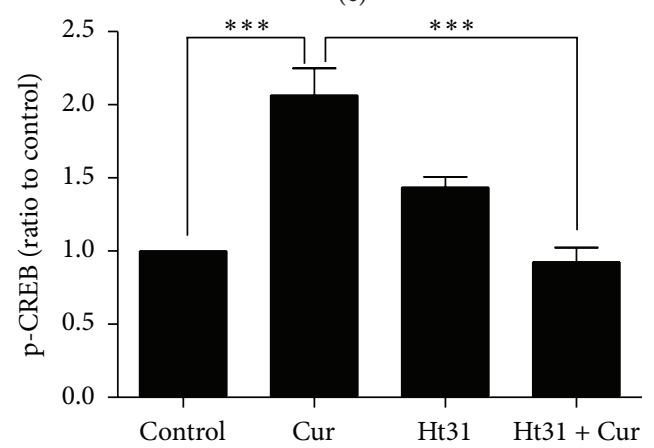

(e)

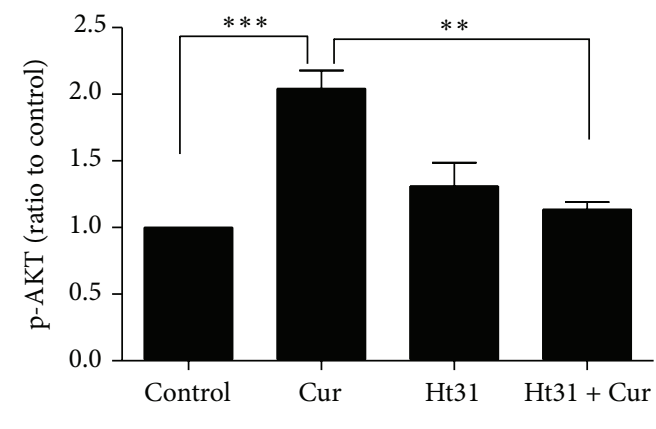

(b)

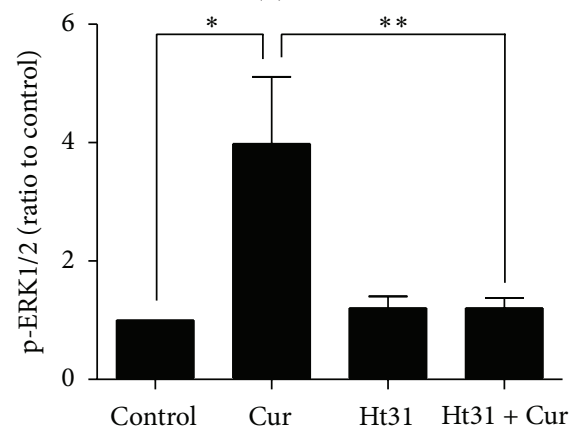

(d)

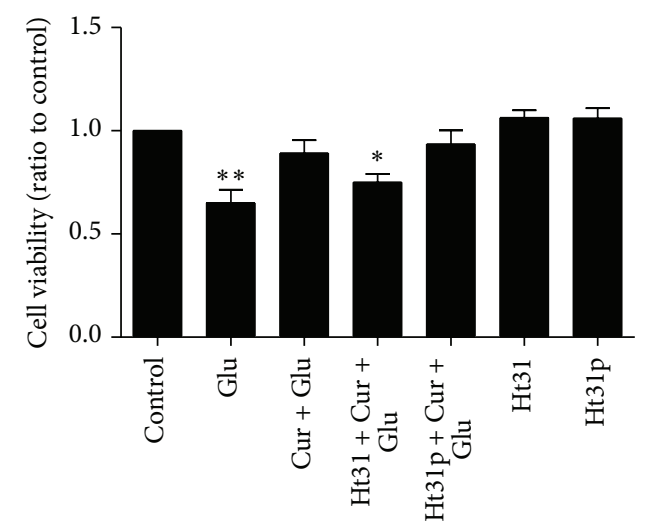

(f)

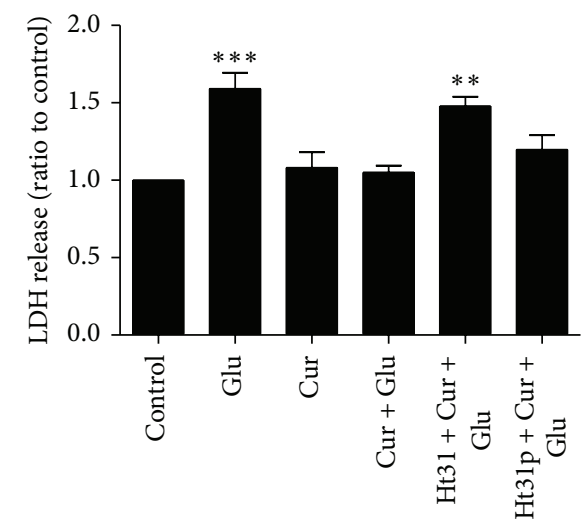

(g)

FIGURE 4: The neuroprotection of curcumin was abolished by PKA anchoring inhibitor Ht31. (a) Western blot analysis of the phosphorylations of AKT, MEK1/2, ERK1/2, and CREB. (b-e) Western blotting bands were quantified using Image J software. (f) Pretreatment with Ht31 suppressed curcumin-mediated increase of cell viability against glutamate insult. (g) LDH assay further confirmed the connection between PKA anchoring and curcumin-mediated neuroprotective effects. ${ }^{*} P<0.05,{ }^{* *} P<0.01$, and ${ }^{* * *} P<0.001$ versus curcumin group (b-e) and versus control values (f, g). $(n=3)$. 


\section{CREB: cAMP response element-binding protein \\ CRE: $\quad$ cAMP response elements \\ LDH: Lactate dehydrogenase \\ MTS: 3-(4,5-Dimethylthiazol-2-yl)-5-(3- \\ carboxymethoxyphenyl)-2-(4- \\ sulfophenyl)-2H-tetrazolium \\ MEK: Mitogen-activated protein kinase \\ ERK: $\quad$ Extracellular regulated kinase \\ ANOVA: Analysis of variance.}

\section{Conflict of Interests}

There is no conflict of interests.

\section{Acknowledgments}

This work was supported by the National Natural Science Foundation of China (nos. 81020108031, 81473235, 91129727, 30973558, 30901815, and 30901803) and Research Fund from Ministry of Education of China (111 Projects no. B07001).

\section{References}

[1] C. M. Dording, D. Mischoulon, T. J. Petersen et al., "The pharmacologic management of SSRI-induced side effects: a survey of psychiatrists," Annals of Clinical Psychiatry, vol. 14, no. 3, pp. 143-147, 2002.

[2] A. E. Autry, M. Adachi, E. Nosyreva et al., "NMDA receptor blockade at rest triggers rapid behavioural antidepressant responses," Nature, vol. 475, no. 7354, pp. 91-95, 2011.

[3] H. Koike, M. Iijima, and S. Chaki, "Involvement of AMPA receptor in both the rapid and sustained antidepressant-like effects of ketamine in animal models of depression," Behavioural Brain Research, vol. 224, no. 1, pp. 107-111, 2011.

[4] M. Gao, W. C. Zhang, Q. S. Liu, J. J. Hu, G. T. Liu, and G. H. Du, "Pinocembrin prevents glutamate-induced apoptosis in $\mathrm{SH}$ SY5Y neuronal cells via decrease of bax/bcl-2 ratio," European Journal of Pharmacology, vol. 591, no. 1-3, pp. 73-79, 2008.

[5] S. Amano, M. Ohashi, T. Ishiko, and F. Hazama, "Humoral factors derived from glial cells protect cultured neuroblastoma cells against glutamate toxicity," Brain Research, vol. 645, no. 1-2, pp. 347-350, 1994.

[6] J. M. May, L. Li, K. Hayslett, and Z.-C. Qu, "Ascorbate transport and recycling by SH-SY5Y neuroblastoma cells: response to glutamate toxicity," Neurochemical Research, vol. 31, no. 6, pp. 785-794, 2006.

[7] G. Z. Réus, R. B. Stringari, K. F. Ribeiro et al., "Ketamine plus imipramine treatment induces antidepressant-like behavior and increases CREB and BDNF protein levels and PKA and PKC phosphorylation in rat brain," Behavioural Brain Research, vol. 221, no. 1, pp. 166-171, 2011.

[8] R. C. Shelton, D. Hal Manier, and D. A. Lewis, "Protein kinases $\mathrm{A}$ and $\mathrm{C}$ in post-mortem prefrontal cortex from persons with major depression and normal controls," The International Journal of Neuropsychopharmacology, vol. 12, no. 9, pp. 12231232, 2009.

[9] P. Brański, A. Palucha, B. Szewczyk, J. M. Wierońska, A. Pilc, and G. Nowak, "Antidepressant-like activity of 8-Br-cAMP, a PKA activator, in the forced swim test," Journal of Neural Transmission, vol. 115, no. 6, pp. 829-830, 2008.

[10] J.-H. Liu, Z.-F. Wu, J. Sun, L. Jiang, S. Jiang, and W.-B. Fu, "Role of AC-cAMP-PKA cascade in antidepressant action of electroacupuncture treatment in rats," Evidence-Based Complementary and Alternative Medicine, vol. 2012, Article ID 932414, 7 pages, 2012.

[11] R. Wang, Y.-B. Li, Y.-H. Li, Y. Xu, H.-L. Wu, and X.-J. Li, "Curcumin protects against glutamate excitotoxicity in rat cerebral cortical neurons by increasing brain-derived neurotrophic factor level and activating TrkB," Brain Research, vol. 1210, pp. 84-91, 2008.

[12] H. Jiang, Z. Wang, Y. Wang et al., "Antidepressant-like effects of curcumin in chronic mild stress of rats: involvement of its antiinflammatory action," Progress in Neuro-Psychopharmacology and Biological Psychiatry, vol. 47, pp. 33-39, 2013.

[13] C. Lu and M. P. Mattson, "Dimethyl sulfoxide suppresses NMDA- and AMPA-induced ion currents and calcium influx and protects against excitotoxic death in hippocampal neurons," Experimental Neurology, vol. 170, no. 1, pp. 180-185, 2001.

[14] N. A. Tsvyetlynska, R. H. Hill, and S. Grillner, "Role of AMPA receptor desensitization and the side effects of a DMSO vehicle on reticulospinal EPSPs and locomotor activity," Journal of Neurophysiology, vol. 94, no. 6, pp. 3951-3960, 2005.

[15] D. Willoughby, W. Wong, J. Schaack, J. D. Scott, and D. M. F. Cooper, "An anchored PKA and PDE4 complex regulates subplasmalemmal cAMP dynamics," The EMBO Journal, vol. 25, no. 10, pp. 2051-2061, 2006.

[16] K. D. Tsai, J. C. Lin, S. M. Yang et al., "Curcumin protects against UVB-induced skin cancers in SKH-1 hairless mouse: analysis of early molecular markers in carcinogenesis," Evidence-Based Complementary and Alternative Medicine, vol. 2012, Article ID 593952, 11 pages, 2012.

[17] Y. Hu, J. Li, P. Liu et al., "Protection of SH-SY5Y neuronal cells from glutamate-induced apoptosis by $3,6^{\prime}$-disinapoyl sucrose, a bioactive compound isolated from radix polygala," Journal of Biomedicine and Biotechnology, vol. 2012, Article ID 728342, 5 pages, 2012.

[18] Z.-W. Sun, L. Zhang, S.-J. Zhu, W.-C. Chen, and B. Mei, "Excitotoxicity effects of glutamate on human neuroblastoma SH-SY5Y cells via oxidative damage," Neuroscience Bulletin, vol. 26, no. 1, pp. 8-16, 2010.

[19] J. Y. Koh and D. W. Choi, "Quantitative determination of glutamate mediated cortical neuronal injury in cell culture by lactate dehydrogenase efflux assay," Journal of Neuroscience Methods, vol. 20, no. 1, pp. 83-90, 1987.

[20] K. E. Smith, E. S. Gibson, and M. L. Dell'Acqua, "cAMPdependent protein kinase postsynaptic localization regulated by NMDA receptor activation through translocation of an Akinase anchoring protein scaffold protein," Journal of Neuroscience, vol. 26, no. 9, pp. 2391-2402, 2006.

[21] J. W. Olney, D. F. Wozniak, and N. B. Farber, "Excitotoxic neurodegeneration in Alzheimer disease: new hypothesis and new therapeutic strategies," Archives of Neurology, vol. 54, no. 10, pp. 1234-1240, 1997.

[22] M. M. Aarts and M. Tymianski, "Novel treatment of excitotoxicity: targeted disruption of intracellular signalling from glutamate receptors," Biochemical Pharmacology, vol. 66, no. 6, pp. 877-886, 2003.

[23] W. Wong and J. D. Scott, "AKAP signalling complexes: focal points in space and time," Nature Reviews Molecular Cell Biology, vol. 5, no. 12, pp. 959-970, 2004. 
[24] M. L. Dell'Acqua, K. E. Smith, J. A. Gorski, E. A. Horne, E. S. Gibson, and L. L. Gomez, "Regulation of neuronal PKA signaling through AKAP targeting dynamics," European Journal of Cell Biology, vol. 85, no. 7, pp. 627-633, 2006.

[25] M. S. Perkinton, T. S. Sihra, and R. J. Williams, " $\mathrm{Ca}^{2+}$-permeable AMPA receptors induce phosphorylation of cAMP response element-binding protein through a phosphatidylinositol 3kinase-dependent stimulation of the mitogen-activated protein kinase signaling cascade in neurons," Journal of Neuroscience, vol. 19, no. 14, pp. 5861-5874, 1999.

[26] B. J. Tunquist, N. Hoshi, E. S. Guire et al., "Loss of AKAP150 perturbs distinct neuronal processes in mice," Proceedings of the National Academy of Sciences of the United States of America, vol. 105, no. 34, pp. 12557-12562, 2008.

[27] P. A. Zunszain, M. A. Horowitz, A. Cattaneo, M. M. Lupi, and C. M. Pariante, "Ketamine: synaptogenesis, immunomodulation and glycogen synthase kinase-3 as underlying mechanisms of its antidepressant properties," Molecular Psychiatry, vol. 18, no. 12, pp. 1236-1241, 2013.

[28] M. Walton, A.-M. Woodgate, A. Muravlev, R. Xu, M. J. During, and M. Dragunow, "CREB phosphorylation promotes nerve cell survival," Journal of Neurochemistry, vol. 73, no. 5, pp. 18361842, 1999.

[29] F. D. Smith, L. K. Langeberg, C. Cellurale et al., "AKAP-Lbc enhances cyclic AMP control of the ERK1/2 cascade," Nature Cell Biology, vol. 12, no. 12, pp. 1242-1249, 2010.

[30] A. Bellis, D. Castaldo, V. Trimarco et al., "Cross-talk between PKA and Akt protects endothelial cells from apoptosis in the late ischemic preconditioning," Arteriosclerosis, Thrombosis, and Vascular Biology, vol. 29, no. 8, pp. 1207-1212, 2009. 


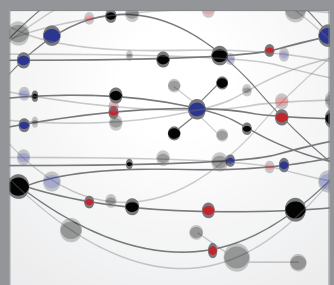

The Scientific World Journal
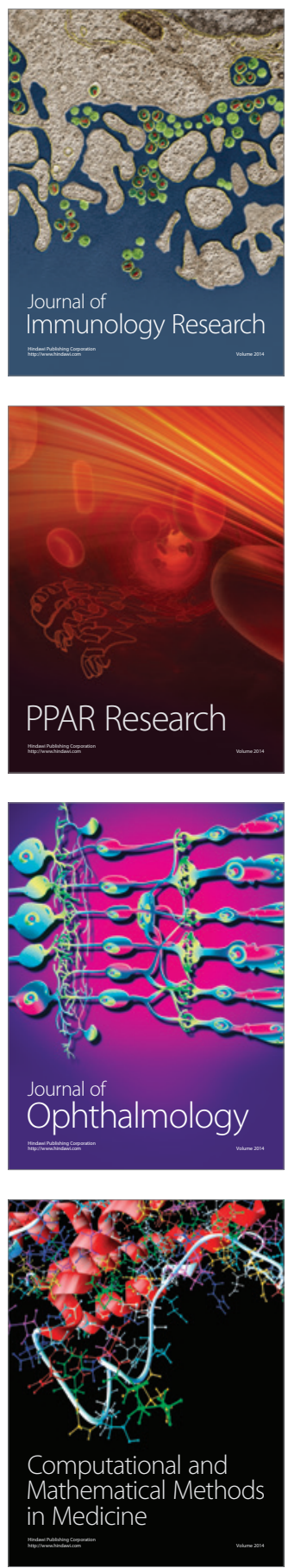

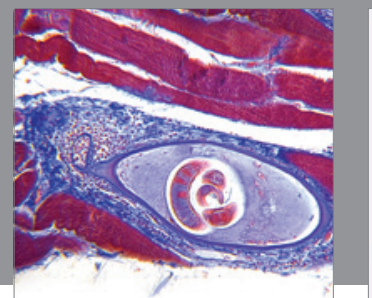

Gastroenterology

Research and Practice
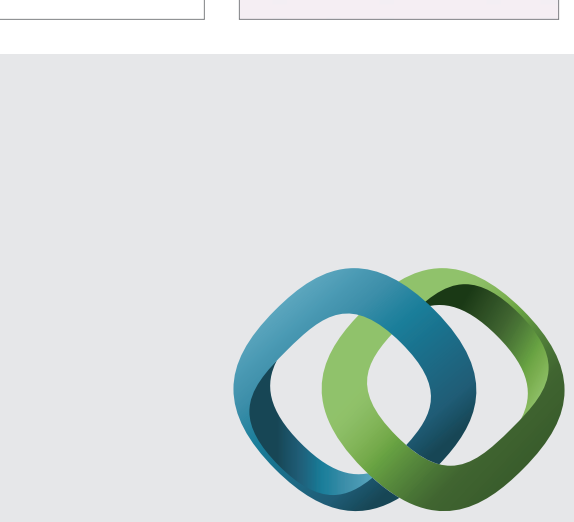

\section{Hindawi}

Submit your manuscripts at

http://www.hindawi.com
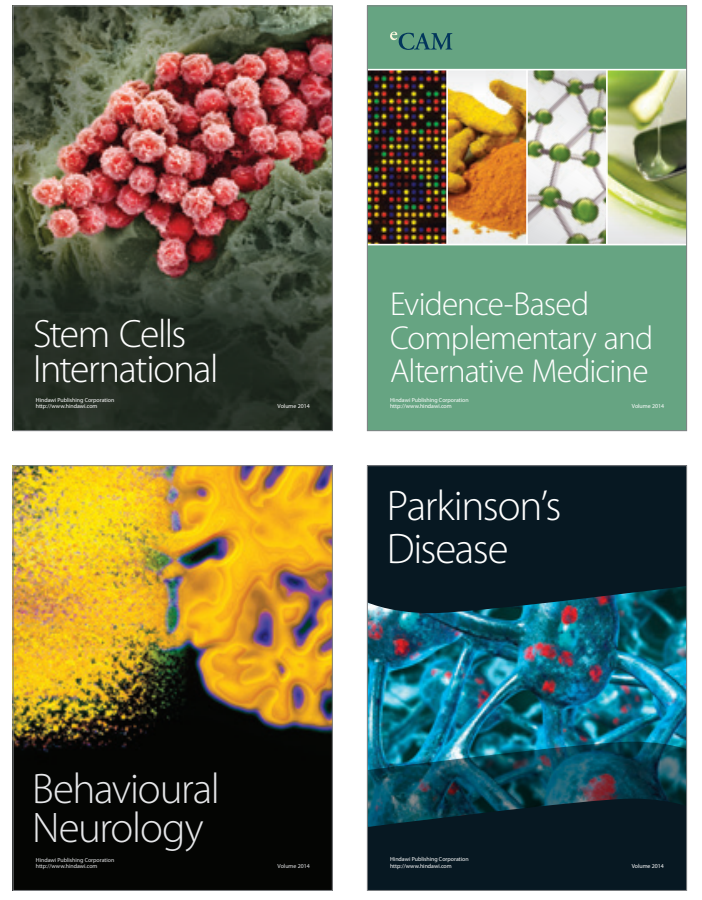
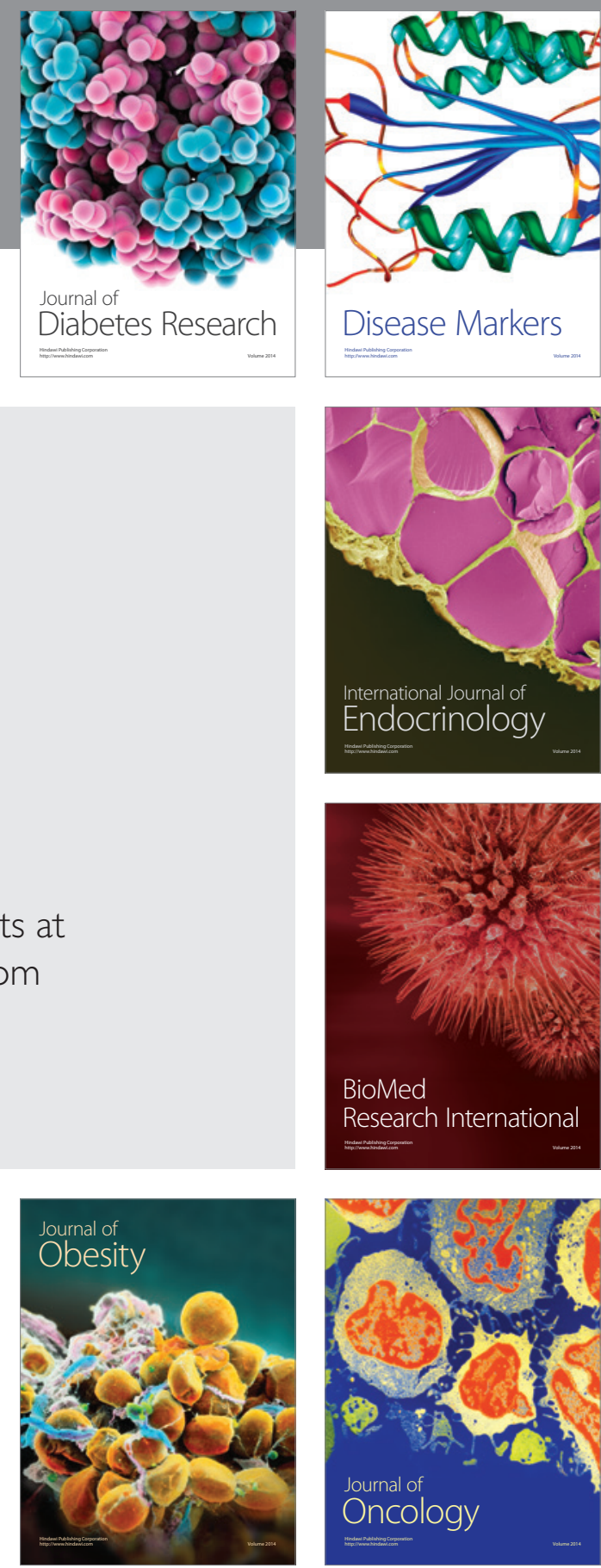

Disease Markers
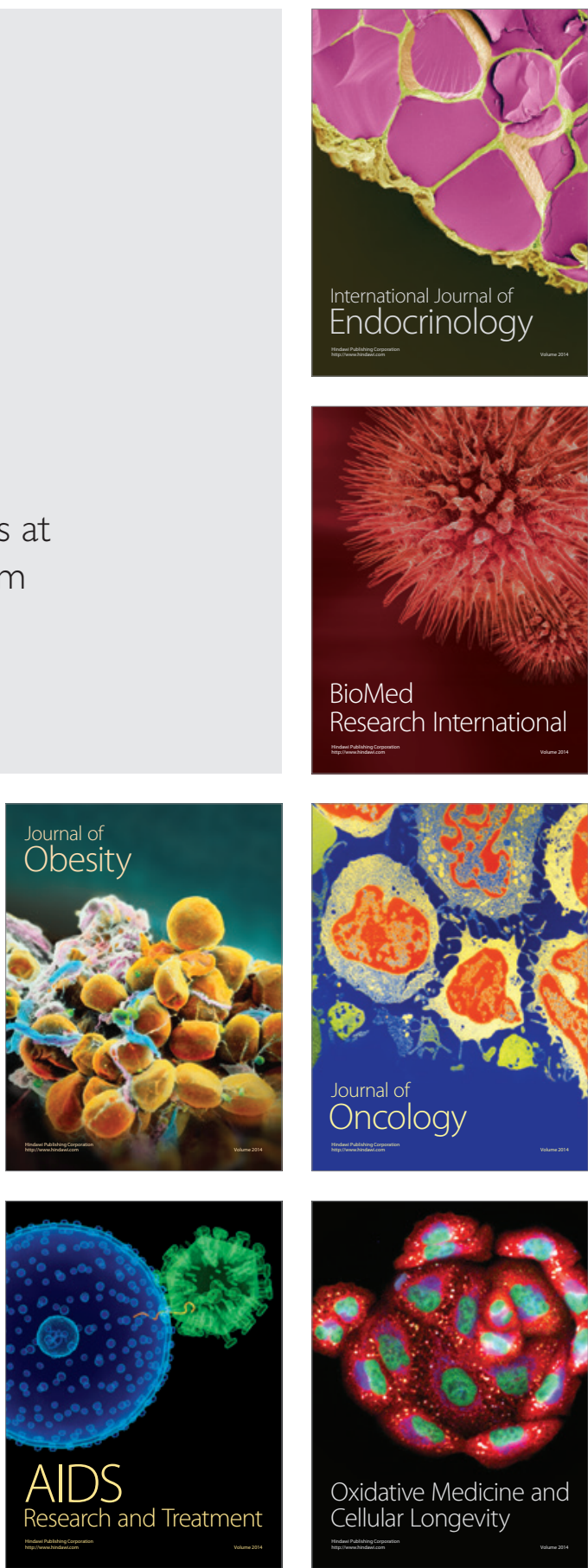